\title{
Multipole methods for nanoantennas design: applications to Yagi-Uda configurations
}

\author{
B. Stout, ${ }^{*}$ A. Devilez, B. Rolly, and N. Bonod \\ Institut Fresnel, Aix-Marseille Université, CNRS, Ecole Centrale Marseille, \\ Domaine Universitaire de St. Jérôme, 13397 Marseille, France \\ ${ }^{*}$ Corresponding author: brian.stout@fresnel.fr
}

Received November 17, 2010; revised February 3, 2011; accepted February 28, 2011; posted March 11, 2011 (Doc. ID 138370); published April 21, 2011

We present a detailed formalism allowing analytical calculations of the radiative properties of nanoantennas. This formalism does not rely on dipole approximations and utilizes multipolar multiple-scattering theory. The improvement in both accuracy and calculation speeds offered by this formulation provides significant advantages that are used in this work to study Yagi-Uda-type nanoantennas. We provide a study that questions the necessity of the reflector particle in nanoantennas. (c) 2011 Optical Society of America

OCIS codes: $\quad 250.5403,290.4210,290.4020,290.5825,160.4236,240.5420$.

\section{INTRODUCTION}

There is an increasing interest in using nanoantennas to extract [ $\underline{1-12}]$ and redirect light [13-23] from quantum emitters such as single molecule fluorophores and quantum dots. Although the emission processes under consideration are intrinsically quantum phenomena, the decay rates of a quantum system depend on the modifications of the local electromagnetic environment induced by the antenna structure. Given that the structures of typical optical antennas, although small, are generally large with respect to the atomic scale (nanometer to micrometer scales overall), semiclassical electromagnetic calculations are usually the most convenient method available for calculating modifications to decay rates and radiation patterns.

In semiclassical calculations, the quantum emitter is replaced by a radiating dipole, and one calculates the modifications to the emitted power, radiated power, and radiation distribution that are induced by the antenna structure. These properties in turn allow one to deduce the modifications to the decay rate and radiation pattern of a quantum emitter. Such calculations require precise information about both near and far fields and generally tend to be quite time consuming, even for modern computers. A notable exception to the previous statement are Mie-type calculations of quantum emission properties in the neighborhood of a single spherical particle. In that case, the multipole approach of the Mie theory allows one to formulate the physical quantities of interest in terms of analytical formulas, which are both highly accurate and rapid to calculate [24-27].

Generalized Mie theory [28-30] has well-established advantages for studying the scattering by multiple-particle systems, including orders of magnitude reductions in computation times. Nevertheless, even in the mutlipole context, the calculation of nanoantenna properties previously required laborious numerical integrations of the Poynting vector in both the near and far field. In this work, we derive multipole formulas for analytically evaluating theses integrals when a quantum emitter is placed in the neighborhood of an antenna structure. Although such multiple-scattering techniques can be applied to systems in which the antenna components are nonspherical [31], in this work, we restrict ourselves to examples of antennas with spherical components where they can be most readily applied.

In the interest of completeness, Section $\underline{2}$ presents a brief overview of the Green function and multiple-scattering formulations for treating electromagnetic sources and scattering. Exact multipole formulas for decay rate enhancements and directivity are derived in Section 3 .

Despite their accuracy, the complexity of multipole techniques can make it difficult to analyze their physical content. Modeling the antenna particles as induced dipoles allows more intuitive physics and, in certain situations, can provide relatively good estimations of the electromagnetic response, provided that the radiation damping effects are accurately modeled. An alternative coupled dipole formulation incorporating new analytic formulas for the radiative properties of nanoantennas is formulated in Section $\underline{4}$.

In Section 5, we use these multipolar and dipolar formulations to study the influence of the number of particles and the presence of reflector particles in nanoantenna designs of the Yagi-Uda type. More precisely, we show that the negative role of the reflector particle on both radiation properties and quantum efficiencies is not always outweighed by gains in directivity.

\section{GREEN FUNCTION FORMULATION}

Given the large quantity and variety of physical information that one wishes to treat during antenna calculations, it proves advantageous to formulate the problem in terms of Green functions. The complexity of the antenna Green function is the chief obstacle to such calculations. We see below and in Section 3, however, that the multipole formulation makes this problem tractable.

A fundamental assumption of this work (which can be relaxed) is that the quantum emitter is sufficiently small with respect to both the antenna structure and the field variations 
to be approximated as a point dipole. The antenna is considered to consist of particles immersed in a homogeneous medium described by a (possibly complex) relative dielectric function $\varepsilon_{b}(\omega)$. With these assumptions, we model the currents in the quantum emitter, $\mathbf{j}_{e}$, by a time harmonic point dipole with strength $\mathbf{p}_{e}$, located at a point $\mathbf{x}_{e}$ within the homogeneous media. The polarization current for such a point dipole is given by $\mathbf{j}_{e}(\mathbf{x})=-i \omega \mathbf{p}_{e} \delta_{\leftrightarrow}^{3}\left(\mathbf{x}-\mathbf{x}_{e}\right)$.

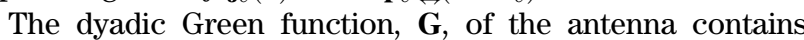
the information necessary for electromagnetic calculations in that it yields the electric field everywhere via the integral formula [28]

$$
\mathbf{E}(\mathbf{x})=i \omega \mu_{0} \int \mathrm{d} \mathbf{x}^{\prime} \stackrel{\leftrightarrow}{\mathbf{G}}\left(\mathbf{x}-\mathbf{x}^{\prime}\right) \mathbf{j}_{e}\left(\mathbf{x}^{\prime}\right)
$$

We simplify matters by restricting $\stackrel{\leftrightarrow}{\mathbf{G}}\left(\mathbf{x}-\mathbf{x}^{\prime}\right)$ to situations where both the source current positions, $\mathbf{x}^{\prime}$, and "receptor" positions, $\mathbf{x}$, are located within the host medium. The Green function can then be separated into an "unperturbed" Green function of the homogeneous exterior medium Green function, $\overleftrightarrow{\mathbf{G}}_{0}$, plus a "scattering" contribution, $\stackrel{\mathbf{G}}{s}_{[}[28,32]$ :

$$
\stackrel{\leftrightarrow}{\mathbf{G}}\left(\mathbf{x}, \mathbf{x}^{\prime}\right)=\stackrel{\leftrightarrow}{\mathbf{G}}_{0}\left(\mathbf{x}, \mathbf{x}^{\prime}\right)+\stackrel{\leftrightarrow}{\mathbf{G}}_{s}\left(\mathbf{x}, \mathbf{x}^{\prime}\right)
$$

The homogeneous unperturbed Green function is translationally invariant and satisfies the equation

$$
\nabla \times \nabla \times \stackrel{\leftrightarrow}{\mathbf{G}}_{0}\left(\mathbf{x}-\mathbf{x}^{\prime}\right)-k_{b}^{2} \stackrel{\leftrightarrow}{\mathbf{G}}_{0}\left(\mathbf{x}-\mathbf{x}^{\prime}\right)=\stackrel{\leftrightarrow}{\mathbb{I}} \delta^{3}\left(\mathbf{x}-\mathbf{x}^{\prime}\right)
$$

where $k_{b}=(\omega / c) \sqrt{\varepsilon_{b}}$ is the wavenumber of the exterior medium. A technical difficulty is that $\stackrel{\leftrightarrow}{\mathbf{G}}_{0}$ is singular at the origin, but this has been studied in detail and one can show that $\stackrel{\leftrightarrow}{\mathbf{G}}_{0}$ can be well defined provided that we treat it as a distribution and take care in treating the limit $\mathbf{x} \rightarrow \mathbf{x}^{\prime}$ [28]. Expressing $\stackrel{\leftrightarrow}{\mathbf{G}}_{0}$ in direct space with $\mathbf{r} \equiv \mathbf{x}-\mathbf{x}^{\prime}$, one has $[28, \overline{32}]$

$$
\begin{aligned}
\stackrel{\leftrightarrow}{\mathbf{G}}_{0}(\mathbf{r})= & \frac{e^{i k_{b} r}}{4 \pi k_{b}^{2} r^{3}} \mathrm{P} . \mathrm{V} \cdot\left\{\left(1-i k_{b} r\right)(3 \hat{\mathbf{r}} \hat{\mathbf{r}}-\stackrel{\leftrightarrow}{\mathbb{I}})+k_{b}^{2} r^{2}(\stackrel{\leftrightarrow}{\mathbb{I}}-\hat{\mathbf{r}} \hat{\mathbf{r}})\right\} \\
& -\frac{\stackrel{\leftrightarrow}{\mathbb{I}}}{3 k_{b}^{2}} \delta^{3}(\mathbf{r}),
\end{aligned}
$$

where P.V. stands for principal value and $\stackrel{\leftrightarrow}{\mathbb{I}}$ is the unit dyadic. As explained in [28], the three-dimensional (3D) delta function term depends on the exclusion volume chosen for the principal value. The formula presented here corresponds to a principal value chosen as either a spherical or cubic infinitesimal volume around the source point. Replacing the total Green function in Eq. (1) with the homogeneous Green function of Eq. (4) yields the electric field, $\mathbf{E}_{0}$, produced by an isolated point dipole:

$$
\begin{aligned}
\mathbf{E}_{0}(\mathbf{x})= & \frac{e^{i k_{b} r}}{4 \pi \varepsilon_{b} \epsilon_{0} r^{3}}\left\{\left(1-i k_{b} r\right)\left[3 \hat{\mathbf{r}}\left(\hat{\mathbf{r}} \cdot \mathbf{p}_{e}\right)-\mathbf{p}_{e}\right]\right. \\
& \left.+k_{b}^{2} r^{2}\left[\mathbf{p}_{e}-\hat{\mathbf{r}}\left(\hat{\mathbf{r}} \cdot \mathbf{p}_{e}\right)\right]\right\}-\frac{\mathbf{p}_{e}}{3 \varepsilon_{b} \epsilon_{0}} \delta^{3}(\mathbf{r}) .
\end{aligned}
$$

The information coming from the antenna structure is embodied in the scattering part of the total Green function, $\stackrel{\leftrightarrow}{\mathbf{G}}_{s}$. The scattering Green function, $\stackrel{\leftrightarrow}{\mathbf{G}}_{s}$, must take into account the multiple scattering of the emitter radiation from all the $N$ components of the antenna structure. For the purpose of cal- culation, it is advantageous to express the scattering Green function in terms of a multiple-scattering $T$ matrix, which we define in operator notation as

$$
\stackrel{\leftrightarrow}{\mathbf{G}}_{s}=\stackrel{\leftrightarrow}{\mathbf{G}}_{0}\left(\sum_{i=1, j=1}^{N} \stackrel{\leftrightarrow}{\mathbf{T}}^{(i, j)}\right) \stackrel{\leftrightarrow}{\mathbf{G}}_{0}
$$

where $i$ and $j$ are particle labels. The multiple-scattering $T$ matrix in this equation has thus been split into $N^{2}$ operators, $\stackrel{\leftrightarrow}{\mathbf{T}}^{(i, j)}$. From a multiple-scattering viewpoint, one can visualize each $\stackrel{\leftrightarrow}{\mathbf{T}}^{(i, j)}$ as representing all multiple-scattering events in which the first particle encountered by incident radiation is $j$ and the last particle encountered is $i[\underline{29}]$.

The $\stackrel{\leftrightarrow}{\mathbf{T}}^{(i, j)}$ can be manageably calculated in the vector partial wave (VPW) basis:

$\stackrel{\leftrightarrow}{\mathbf{T}}^{(i, j)}=\sum_{q=1, q^{\prime}=1}^{2} \sum_{n=1 ; n^{\prime}=1}^{\infty} \sum_{m=-n}^{m=n} \sum_{m^{\prime}=-n^{\prime}}^{m^{\prime}=n^{\prime}}\left|\Psi_{q, n, m}\right\rangle T_{q, n, m ; q^{\prime}, n^{\prime}, m^{\prime}}^{(i, j)}\left\langle\Psi_{q^{\prime} n^{\prime}, m^{\prime}}\right|$,

where $\left|\Psi_{q, n, m}\right\rangle$ are the VPW states (see Appendix A.2 for explicit representations) and are specified by the three discrete "quantum" numbers: $q=1,2 ; n=1,2, \ldots \infty$; and $m=-n, \ldots, n$ (which for the purpose of numerical computations can be combined into a single discrete index $[\underline{30}, \underline{33}])$. The great advantage of the VPW basis is that the $\stackrel{\leftrightarrow}{\mathbf{T}}^{(i, j)}$ operator is determined as an infinite dimensional matrix in the VPW space that can be calculated from the $T$ matrices of the isolated particles, denoted $t^{(j)}(j=1, \ldots, N)$, and the pairwise translationaddition matrices, $H^{(i, j)}$, between particle centers [see Eq. (8)]. The $T^{(i, j)}$ matrices, henceforth referred to as bodycentered $T$ matrices, can be rendered finite by truncating the orbital quantum numbers, $n$, to some finite dimension, $n_{\max }$ (whose value depends on particle sizes and interaction strengths).

A number of different methods for calculating the $T^{(i, j)}$ matrices exist in the literature (generally formulated within the Foldy-Lax framework [30,33,34]). Probably one of the simplest methods (both conceptually and numerically) is to extract the $T^{(i, j)}$ as the submatrices obtained after inversion of the following system matrix, i.e.,

$$
\begin{gathered}
{\left[\begin{array}{cccc}
T_{N}^{(1,1)} & T_{N}^{(1,2)} & \ldots & T_{N}^{(1, N)} \\
T_{N}^{(2,1)} & T_{N}^{(2,2)} & \ldots & T_{N}^{(2, N)} \\
\vdots & \vdots & \ddots & \vdots \\
T_{N}^{(N, 1)} & T_{N}^{(N, 2)} & \ldots & T_{N}^{(N, N)}
\end{array}\right]} \\
=\left[\begin{array}{cccc}
{\left[t^{(1)}\right]^{-1}} & -H^{(1,2)} & \ldots & -H^{(1, N)} \\
-H^{(2,1)} & {\left[t^{(2)}\right]^{-1}} & \cdots & -H^{(2, N)} \\
\vdots & \vdots & \ddots & \vdots \\
-H^{(N, 1)} & -H^{(N, 2)} & \cdots & {\left[t^{(N)}\right]^{-1}}
\end{array}\right],
\end{gathered}
$$

where $H^{(j, l)} \equiv H\left(k_{b}\left(\mathbf{x}_{j}-\mathbf{x}_{l}\right)\right)$ are irregular translation matrices (analytical expressions for the matrix elements of $H(k \mathbf{x})$ as well as those of the regular translation matrix $J(k \mathbf{x})$ are given in $[\underline{28}, \underline{29}, \underline{35}])$.

It should be noted that, for a long time, such direct matrix inversions were disregarded in 3D calculations due to the fact 
that matrices like the one on the right-hand side of Eq. ( 6 ) were numerically ill conditioned for matrix inversion. However, it has been recently pointed out that such direct matrix inversions work quite well provided that one employs analytical matrix balancing techniques [30]. In lieu of employing matrix balancing, many multiple-scattering codes privilege iterative techniques where the ill-conditioning of the problem can also be handled via conjugate gradient techniques. Although numerically performant, iterative techniques pose the disadvantage of having an additional convergence parameter and not all iterative formulations determine the $T^{(i, j)}$ matrices.

Once the on-shell $T$ matrices have been determined using Eq. (8) or an alternative technique, we can derive analytic expressions for the electromagnetic properties of the antenna system, such as decay rates, local fields, and far-field emission. Notably, the total emitted power is evaluated by time averaging $P_{t} \equiv-\mathbf{E}_{t} \cdot \mathbf{j}_{\text {src }}$ over a period where $\mathbf{E}_{t}$ is the electric field produced by the source current while taking into account interactions with the antenna structure. Averaging this power over a period, $T=2 \pi / \omega$, we obtain [32]

$$
\begin{aligned}
\Gamma_{t} & \equiv-\frac{1}{T} \int_{0}^{T} \mathrm{~d} t \int \mathrm{d} \mathbf{x} \mathbf{E}_{t}(\mathbf{x}, t) \cdot \mathbf{j}_{e}(\mathbf{x}, t) \\
& =\frac{\omega^{3}}{2} \mu_{0} \operatorname{Im}\left\{\mathbf{p}_{e}^{*}\left(\mathbf{x}_{j}\right) \cdot \stackrel{\leftrightarrow}{\mathbf{G}}\left(\mathbf{x}_{j}, \mathbf{x}_{j}\right) \cdot \mathbf{p}_{e}\left(\mathbf{x}_{j}\right)\right\} .
\end{aligned}
$$

Some of the power emanating from the dipole emitter will be dissipated by the antenna. The rest will be radiated off into the far field where it can be detected. The calculation of the radiated power proceeds by first taking the far-field limit of the electric field given by Eq. (1). The electric field is transverse in the far-field limit, and we can readily obtain the $\mathbf{H}$ field from the electric field via the relation [36]

$$
\lim _{r \rightarrow \infty} \mathbf{H}_{t}(\mathbf{r})=\frac{k_{b}}{\mu_{0} \omega} \hat{\mathbf{r}} \times \mathbf{E}_{t}
$$

In the $r \rightarrow \infty$ limit, the time-averaged Poynting vector is thus

$$
r \rightarrow \infty \lim _{r \rightarrow \infty}\langle\mathbf{S}\rangle=\lim _{r \rightarrow \infty} \frac{1}{2} \operatorname{Re}\left\{\mathbf{E}_{t}^{*} \times \mathbf{H}_{t}\right\}=\frac{1}{2} \frac{k_{b}}{\omega \mu_{0}} \hat{\mathbf{r}}\left\|\mathbf{E}_{t}\right\|^{2} .
$$

The far-field irradiance, $I_{r}(\theta, \phi)$, and total radiated power are defined respectively by

$$
I_{r}(\theta, \phi) \equiv \lim _{r \rightarrow \infty} r^{2}\langle\mathbf{S}\rangle \cdot \hat{\mathbf{r}} \quad \text { and } \quad \Gamma_{r} \equiv \int \mathrm{d} \Omega I_{r}(\theta, \phi) .
$$

In order to determine the modifications to $\Gamma_{t}, I_{r}(\theta, \phi)$, and $\Gamma_{r}$ induced by the antenna, we will need to normalize these values with respect to the corresponding quantities of an isolated emitter placed inside the homogeneous background. In this case, the analytical expressions for the field and homogeneous Green function of Eqs. (4) and (ㄷ) yield the textbook results for dipole emission in a homogeneous medium. Notably, the power emitted in a homogeneous dielectric medium is

$$
\begin{aligned}
\Gamma_{t, 0} & =\frac{\omega^{3}}{2} \mu_{0} \operatorname{Im}\left\{\mathbf{p}_{e}^{*}\left(\mathbf{x}_{j}\right) \cdot \stackrel{\leftrightarrow}{\mathbf{G}}_{0}\left(\mathbf{x}_{j}, \mathbf{x}_{j}\right) \cdot \mathbf{p}_{e}\left(\mathbf{x}_{j}\right)\right\} \\
& =\left|\mathbf{p}_{e}\right|^{2} \frac{\omega^{3}}{12 \pi \epsilon_{0} c^{2}} \operatorname{Re}\left\{k_{b}\right\} .
\end{aligned}
$$

The classic far-field radiation pattern and radiated power, $\Gamma_{0}$, are readily obtained from Eq. (5) applied to Eqs. (11) and (12):

$$
\begin{aligned}
I_{r, 0}(\hat{\mathbf{r}}) & =\frac{\omega^{3} k_{b}}{32 \pi^{2} \epsilon_{0} c^{2}}\left(1-\left(\hat{\mathbf{r}} \cdot \hat{\mathbf{p}}_{e}\right)^{2}\right)\left|\mathbf{p}_{e}\right|^{2}, \\
\Gamma_{0} & \equiv \int \mathrm{d} \Omega I_{r, 0}(\theta, \phi)=\left|\mathbf{p}_{e}\right|^{2} \frac{\omega^{3} k_{b}}{12 \pi \epsilon_{0} c^{2}} .
\end{aligned}
$$

This concludes the review of the background material necessary to derive the multipole antenna formulas of Section 3 .

\section{MULTIPOLE FORMULAS FOR DECAY RATE ENHANCEMENTS}

The purpose of this section is to generalize the analytic expressions for emitted and radiated powers to the case in which the emitter is located near a nanoantenna structure. We saw in Section 2 that the multiple-scattering $T$ matrix of Eq. (8) determines the scattering Green function of Eq. (6). The scattering Green function can then be expressed on the VPW basis provided that we also express the homogeneous Green function on the VPW basis. Taking advantage of the translational invariance of $\mathbf{G}_{0}\left(\mathbf{x}, \mathbf{x}^{\prime}\right)$, it can be written as [28]

$$
\stackrel{\leftrightarrow}{\mathbf{G}}_{0}(\mathbf{r}, \mathbf{0})=i k_{b} \sum_{m=-1}^{1} \mathbf{N}_{1 m}\left(k_{b}, \mathbf{r}\right) \operatorname{Rg}\left\{\tilde{\mathbf{N}}_{1 m}(\mathbf{0})\right\}-\frac{\hat{\mathbf{r}} \hat{\mathbf{r}}}{k_{b}^{2}} \delta(\mathbf{r}),
$$

where $\mathbf{N}_{1,-1}, \mathbf{N}_{1,0}$, and $\mathbf{N}_{11}$ are the three outgoing electric dipolar partial wave functions ( $\mathbf{N}$ functions correspond to the $q=2$ VPWs detailed in Appendix A.2). Employing this expression in Eq. (1), the unperturbed electric field created by an isolated point dipole, denoted $\mathbf{E}_{0}$, is then expressed as

$$
\begin{aligned}
\mathbf{E}_{0}(\mathbf{x}) & =\omega^{2} \mu_{0} \int \mathrm{d} \mathbf{x}^{\prime} \stackrel{\leftrightarrow}{\mathbf{G}_{0}}\left(\mathbf{x}, \mathbf{x}^{\prime}\right) \cdot \mathbf{p}_{e} \delta^{3}\left(\mathbf{x}^{\prime}\right) \\
& =\frac{i k_{b} \omega^{2} p_{e}}{\epsilon_{0} c^{2}} \sum_{m=-1}^{1} \mathbf{N}_{1 m}\left(k_{b} \mathbf{x}\right) f_{2,1, m},
\end{aligned}
$$

where we define the outgoing dipole field coefficients, $f_{q=2, n=1, m}$, to be given by

$$
f_{2,1, m} \equiv R g\left\{\tilde{\mathbf{N}}_{1 m}(\mathbf{0})\right\} \cdot \hat{\mathbf{n}}=\left\langle\Psi_{2, n, m} \mid \mathbf{0}\right\rangle \cdot \hat{\mathbf{n}}
$$

where $\hat{\mathbf{n}}$ is the unit vector in the direction of the emitter dipole moment, defined such that $\mathbf{p}_{e}=p_{e} \hat{\mathbf{n}}$. All other emitter multipoles, $n>1$ or $q \neq 2$, are taken to be zero.

Utilizing the analytical expression for $\operatorname{Rg}\left\{\tilde{\mathbf{N}}_{1 m}(\mathbf{0})\right\}$, we obtain the following expressions for the emitter field coefficients:

$$
\begin{aligned}
f_{q, n, 0} & =\delta_{q, 2} \delta_{n, 1} \sqrt{\frac{1}{6 \pi}} \hat{\mathbf{z}} \cdot \hat{\mathbf{n}}, \quad f_{q, n, 1}=\frac{\delta_{q, 2} \delta_{n, 1}}{2 \sqrt{3 \pi}}(-\hat{\mathbf{x}}+i \hat{\mathbf{y}}) \cdot \hat{\mathbf{n}}, \\
f_{q, n,-1} & =\frac{\delta_{q, 2} \delta_{n, 1}}{2 \sqrt{3 \pi}}(\hat{\mathbf{x}}+i \hat{\mathbf{y}}) \cdot \hat{\mathbf{n}} .
\end{aligned}
$$

Employing Eq. (15) for $\stackrel{\leftrightarrow}{\mathbf{G}}_{0}$ in Eqs. (6), (1), and (2) and invoking the translation-addition theorem, we obtain an entirely multipolar expression for the field radiated by a dipole emitter interacting with an antenna structure:

$$
\begin{aligned}
\mathbf{E}_{t}(\mathbf{r})= & \mathbf{E}_{0}+\mathbf{E}_{\mathbf{s}}=\frac{i p_{e} k_{b} \omega^{2}}{\epsilon_{0} c^{2}}[\mathbf{N}(\mathbf{r}) f \\
& \left.+\sum_{j, l=1}^{N}\left[\mathbf{M}\left(k \mathbf{r}_{j}\right), \mathbf{N}\left(k \mathbf{r}_{j}\right)\right] T^{(j, l)} H^{(l, e)} f\right] \equiv \frac{i p_{e} k_{b} \omega^{2}}{\epsilon_{0} c^{2}} \tilde{\mathbf{E}}_{t}(\mathbf{r}),
\end{aligned}
$$


where $f$ denotes a column matrix containing the emitter coefficients in the multipole space (with only electric dipole elements nonzero) and $H^{(l, e)} \equiv H\left(k_{b}\left(\mathbf{x}_{l}-\mathbf{x}_{e}\right)\right)$ are the irregular translation-addition matrices between the position of particle $l$ and the emitter position.

In the second line of Eq. (19), we defined a dimensionless field, $\tilde{\mathbf{E}}_{t}$, proportional to the total electric field. This definition of $\tilde{\mathbf{E}}_{t}$ proves convenient when normalizing the antenna irradiance, $I_{r}(\theta, \phi)$, with respect to $\Gamma_{0} /(4 \pi)$ of the isolated emitter. Using the definition of Eq. (19) in Eqs. (11) and (12), the normalized irradiance is given by

$$
\tilde{I}_{r} \equiv \frac{4 \pi I_{r}(\theta, \phi)}{\Gamma_{0}}=24 \pi^{2} \lim _{r \rightarrow \infty}\left(k_{b} r\right)^{2}\left\|\tilde{\mathbf{E}}_{t}(\mathbf{r})\right\|^{2} .
$$

The electric field of Eq. (19) can then be utilized in Eq. (9) to obtain the decay rate enhancement factor (valid even in an absorbing host medium):

$$
\tilde{\Gamma}_{t} \equiv \frac{\Gamma_{t}}{\Gamma_{t}, 0}=1+\frac{\operatorname{Re}\left\{6 \pi k_{b} \sum_{j, l=1}^{N} f^{\dagger} H^{(e, j)} T^{(j, l)} H^{(l, e)} f\right\}}{\operatorname{Re}\left\{k_{b}\right\}} .
$$

Likewise, for an absorption-free host medium, the enhancement in radiative decay rate is obtained by inserting Eq. (19) into Eqs. (11) and (12). Utilizing the translation-addition theorem and the orthogonality properties of the vector spherical harmonics (VSHs), one obtains for the radiative decay rate enhancement

$$
\begin{aligned}
\tilde{\Gamma}_{r} \equiv \frac{\Gamma_{r}}{\Gamma_{0}}= & 1+6 \pi \sum_{i, j, k, l=1}^{N}\left[T^{(j, i)} H^{(i, e)} f\right]^{\dagger} J^{(j, k)} T^{(k, l)} H^{(l, e)} f \\
& +12 \pi \operatorname{Re}\left[\sum_{j, l=1}^{N} f^{\dagger} J^{(e, j)} T^{(j, l)} H^{(l, e)} f\right]
\end{aligned}
$$

where we see that Eqs. (21) and (22) required the use of both regular, $J$, and irregular, $H$, translation-addition matrices $[28,29,35]$.

The multiple-scattering results of Eqs. (21) and (22) simplify considerably when a single antenna particle is present:

$$
\begin{gathered}
\tilde{\Gamma}_{t}=1+\frac{\operatorname{Re}\left\{6 \pi k_{b} f^{\dagger} H^{(e, j)} t H^{(j, e)} f\right\}}{\operatorname{Re}\left\{k_{b}\right\}}, \\
\tilde{\Gamma}_{r}=1+6 \pi\left[H^{(j, e)} f\right]^{\dagger} t^{\dagger} t H^{(j, e)} f+12 \pi \operatorname{Re}\left[f^{\dagger} J^{(e, j)} t H^{(j, e)} f\right],
\end{gathered}
$$

where $t$ is the single-particle $T$ matrix. If the $T$ matrix is that of a spherical (Mie) scatterer, then Eqs. (23) and (24) are equivalent to expressions that were derived previously for Mie scatterers [24-27].

\section{INDUCED DIPOLE FORMULATION FOR YAGI-UDA DESIGNS}

In the Yagi-Uda-type designs considered in Section $\underline{5}$, the emitting dipole and spherical antenna elements are all positioned along the same axis, henceforth denoted the $x$ axis [see Fig. 1(a)]. We furthermore consider the emitting dipole to be perpendicular to the $x$ axis, henceforth denoted the $z$ axis. For sufficiently small antenna particles and sufficiently large separations, it is possible for electric dipole excitations to dominate the antenna response. Dipole couplings have been

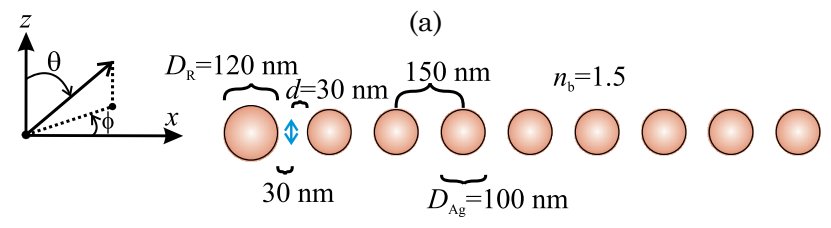

(b)

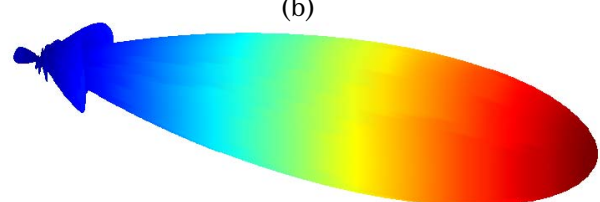

Fig. 1. (Color online) (a) Yagi-Uda antenna design with a silver reflector element $120 \mathrm{~nm}$ in diameter and a collector array of eight silver spheres $100 \mathrm{~nm}$ in diameter. (b) Radiation pattern $\tilde{I}(\theta, \phi)$ of this antenna in the far field for an emitter operating at a vacuum wavelength of $\lambda_{v}=618 \mathrm{~nm}$.

widely studied and can lead to more intuitive physics than multipole couplings. Therefore, in this section, we derive coupled dipole analogs to Eqs. (21) and (22) for the antenna geometries studied in Section $\underline{5}$ in order to compare with multipole calculations.

\section{A. Coupled Dipole Formalism}

We follow the terminology of dielectric polarizability usually employed when dealing with dipole approximations. In this context, the dipole moment induced in a material particle immersed in a material medium of relative dielectric constant $\varepsilon_{b}$ is proportional to the excitation field via the relation

$$
\mathbf{p}(\omega)=\epsilon_{0} \varepsilon_{b} \alpha(\omega) \mathbf{E}_{\mathrm{exc}}(\omega),
$$

where $\alpha(\omega)$ is the frequency-dependent polarizability characterizing the particle.

We denote the field strength of an arbitrary $\hat{\mathbf{z}}$-polarized "incident" field at each particle position as $\mathbf{E}_{\mathrm{inc}, j}=E_{\mathrm{inc}, j} \hat{\mathbf{z}}$ and the excitation fields as $\mathbf{E}_{\mathrm{exc}, j}=E_{\mathrm{exc}, j} \hat{\mathbf{z}}$. Using the dipole field expression of Eq. (5), the excitation field of the $j^{\text {th }}$ particle in the finite chain of coupled dipoles can then be written as

$$
\begin{aligned}
E_{\mathrm{exc}, j} & =E_{\mathrm{inc}, j}-\sum_{l \neq j} e^{i k_{b} d_{j, l}} \frac{a_{l}^{3}}{d_{j, l}^{3}}\left(1-i k_{b} d_{j, l}-k_{b}^{2} d_{j, l}^{2}\right) \tilde{\alpha}_{l}(\omega) E_{\mathrm{exc}, l} \\
& =E_{\mathrm{inc}, j}+\sum_{l \neq j} \gamma_{j, l} \tilde{\alpha}_{l}(\omega) E_{\mathrm{exc}, l},
\end{aligned}
$$

where, in order to simplify the notation, we defined dimensionless "polarizability" functions $\tilde{\alpha}_{j}(\omega)$ as

$$
\tilde{\alpha}_{j}(\omega) \equiv \frac{\alpha_{j}(\omega)}{4 \pi a_{j}^{3}}
$$

with $a_{j}$ as the radius of the $j^{\text {th }}$ sphere. We also define, for Eq. (26) and formulas below, dimensionless field coupling factors, $\gamma_{j, l}$, between particles $l$ and $j$ and couplings, $\gamma_{j, e}$, between the emitter and a particle $j$ as

$$
\begin{aligned}
\gamma_{j, l} & \equiv-e^{i k_{b} d_{j, l}}\left(\frac{a_{l}}{d_{j, l}}\right)^{3}\left(1-i k_{b} d_{j, l}-k_{b}^{2} d_{j, l}^{2}\right) \\
\gamma_{j, e} & \equiv-e^{i k_{b} d_{j, e}}\left(\frac{a_{j}}{d_{j, e}}\right)^{3}\left(1-i k_{b} d_{j, e}-k_{b}^{2} d_{j, e}^{2}\right)
\end{aligned}
$$


where $d_{j, l} \equiv\left|x_{j}-x_{l}\right|$ and $d_{j, e} \equiv\left|x_{j}-x_{e}\right|$ are the distances between different particle centers and the distance of particles from the emitter, respectively. field

Also, defining quantities $\tilde{p}_{j}$ with the dimension of electric

$$
\tilde{p}_{j} \equiv \tilde{\alpha}_{j}(\omega) E_{\mathrm{exc}, j}
$$

the coupled equations in Eq. (26) can then be solved via matrix inversion:

$$
\left[\begin{array}{c}
\tilde{p}_{1} \\
\vdots \\
\tilde{p}_{N}
\end{array}\right]=\left[\begin{array}{cccc}
\tilde{\alpha}_{1}^{-1} & -\gamma_{1,2} & \cdots & -\gamma_{1, N} \\
-\gamma_{2,1} & \tilde{\alpha}_{2}^{-1} & \cdots & -\gamma_{2, N} \\
\vdots & \cdots & \ddots & \vdots \\
-\gamma_{N, 1} & -\gamma_{N, 2} & \cdots & \tilde{\alpha}_{N}^{-1}
\end{array}\right]^{-1}\left[\begin{array}{c}
E_{\mathrm{inc}, 1} \\
\vdots \\
E_{\mathrm{inc}, N}
\end{array}\right] .
$$

The matrix inversion in Eq. (30) is the dipole analog of the multipole $T$-matrix evaluation of Eq. (8). One can next define "effective" dimensionless polarizabilities, $\tilde{\alpha}_{j}^{\text {eff }}$, that contain all multiple-scattering effects as

$$
\tilde{\alpha}_{j}^{\mathrm{eff}}(\omega) \equiv \frac{\tilde{p}_{j}}{E_{\mathrm{inc}, j}}
$$

The field incident on each particle from the dipolar emitter in the absence of an antenna is

$$
E_{\mathrm{inc}, j}=-e^{i k_{b} d_{j, e}}\left(\frac{1}{d_{j, e}}\right)^{3}\left(1-i k_{b} d_{j, e}-k_{b}^{2} d_{j, e}^{2}\right) \frac{p_{\mathrm{e}}}{4 \pi \epsilon_{0} \varepsilon_{b}} .
$$

Once the $\tilde{\alpha}_{j}^{\text {eff }}$ of Eq. (31) have been calculated, the total electric field can be written as

$$
\begin{aligned}
\mathbf{E}_{t}(\mathbf{r})= & \frac{1}{4 \pi \epsilon_{0} \varepsilon_{b}} \sum_{j=0}^{N} \frac{e^{i k_{b} r_{j}}}{r_{j}^{3}}\left\{\left(1-i k_{b} r_{j}\right)\left[3 \hat{\mathbf{r}}_{j}\left(\hat{\mathbf{r}}_{j} \cdot \mathbf{p}_{j}\right)-\mathbf{p}_{j}\right]\right. \\
& \left.+k_{b}^{2} r_{j}^{2}\left(\mathbf{p}_{j}-\hat{\mathbf{r}}_{j}\left(\hat{\mathbf{r}}_{j} \cdot \mathbf{p}_{j}\right)\right)\right\},
\end{aligned}
$$

where $\mathbf{r}_{j}=r_{j} \hat{\mathbf{r}}_{j} \equiv \mathbf{r}-\mathbf{x}_{j}$ are the relative positions with respect to the particle centers, $\mathbf{x}_{j}$, with $j=0$ designating the dipole emitter (i.e., $\mathbf{x}_{0} \equiv \mathbf{x}_{e}$ and $\mathbf{p}_{0} \equiv \mathbf{p}_{e}$ ).

Utilizing the expressions of Eqs. (31)-(33) and invoking the definitions of dimensionless fields and intensities of Eqs. (19) and (20), we obtain relatively simple expressions for the far field and irradiance:

$$
\begin{aligned}
\lim _{r \rightarrow \infty} \tilde{\mathbf{E}}_{t}(r, \theta, \phi) & =\frac{e^{i k_{b} r}}{4 \pi i k_{b} r} \sin \theta \hat{\theta}\left[1+\sum_{j=1}^{N} \gamma_{j, e} \tilde{\alpha}_{j}^{\mathrm{eff}} e^{-i k_{b} d_{j, e} \sin \theta \cos \phi}\right] \\
\tilde{I}_{r}(\theta, \phi) & \equiv \frac{4 \pi I_{r}(\theta, \phi)}{\Gamma_{0}} \\
& =\frac{3}{2} \sin ^{2} \theta \mid 1+\sum_{j=1}^{N} \gamma_{j, e} \tilde{\alpha}_{j}^{\mathrm{eff}} e^{-\left.i k_{b} d_{j, e} \sin \theta \cos \phi\right|^{2},}
\end{aligned}
$$

where $\theta$ and $\phi$ are the angles with respect to the $z$ and $x$ axes, respectively.

As with the multipole approach, the enhancement in the radiative decay rate is obtained by inserting Eq. (33) into Eqs. (11) and (12). We can calculate emission and radiative enhancement factors in a manner analogous to that carried out in Section 3. After some algebra and integrations, we obtain the following formulas for the dipolar enhancement factors:

$$
\begin{gathered}
\tilde{\Gamma}_{t} \equiv \frac{\Gamma_{t}}{\Gamma_{t, 0}}=1+\frac{3}{2} \sum_{j=1}^{N} \frac{\operatorname{Im}\left\{\left(\frac{\gamma_{j, e}}{k_{b} a_{j}}\right)^{2} \tilde{\alpha}_{j}^{\text {eff }}\right\}}{\operatorname{Re}\left\{k_{b} a_{j}\right\}}, \\
\tilde{\Gamma}_{r} \equiv \frac{\Gamma_{r}}{\Gamma_{0}}=1+\sum_{j=1}^{N}\left|\tilde{\alpha}_{j}^{\text {eff }} \gamma_{j, e}\right|^{2}+\sum_{j=1}^{N} 3 \operatorname{Re}\left[\tilde{\alpha}_{j}^{\text {eff }} \gamma_{j, e}\right] \\
\times\left[\frac{\left(k_{b} d_{j, e}\right)^{2}-1}{\left(k_{b} d_{j, e}\right)^{3}} \sin \left(k_{b} d_{j, e}\right)+\frac{\cos \left(k_{b} d_{j, e}\right)}{\left(k_{b} d_{j, e}\right)^{2}}\right] \\
+\sum_{l>j}^{N} 3 \operatorname{Re}\left[\gamma_{l, e}^{*} \tilde{\alpha}_{l}^{\text {eff }, *} \tilde{\alpha}_{j}^{\text {eff }} \gamma_{j, e}\right] \\
\times\left[\frac{\left(k_{b} d_{l, j}\right)^{2}-1}{\left(k_{b} d_{l, j}\right)^{3}} \sin \left(k_{b} d_{l, j}\right)+\frac{\cos \left(k_{b} d_{l, j}\right)}{\left(k_{b} d_{l, j}\right)^{2}}\right],
\end{gathered}
$$

which are, respectively, the electric dipole analogs of Eqs. (21) and (22).

\section{B. Time Harmonic Polarizability}

To evaluate the formulas in Eqs. (35) and (36), we need an accurate model of the frequency-dependent dipole polarizability $\alpha(\omega)$ of the plasmonic antenna components. The commonly adopted quasi-static approximation or even the more sophisticated pointlike models [37-39] can introduce inaccuracies associated with the model rather than actual defaults of the dipole approximation per se. To avoid such problems, we adopted the unconventional choice of using the dipole polarizability prescribed by Mie theory.

Taking into account the differences in formalism between the multipole framework and the polarizability picture, we find that the electric dipole polarizability of a sphere is

$$
\alpha(\omega)=\frac{6 \pi}{i k_{b}^{3}} t_{2,1}(\omega),
$$

where $t_{q=2, n=1}(\omega)$ is the electric dipole element of the (diagonal) Mie $T$ matrix[36] (nondependent on $m$ due to spherical symmetry). This gives us a fully analytic frequency-dependent polarizability corresponding to the electric dipolar response from Mie theory:

$$
\tilde{\alpha}(\omega)=\frac{\alpha(\omega)}{4 \pi a^{3}}=\frac{3}{2 i\left(a k_{b}\right)^{3}} \frac{j_{1}\left(a k_{b}\right)}{h_{1}\left(a k_{b}\right)} \frac{\varepsilon_{s} \varphi_{1}\left(a k_{b}\right)-\varepsilon_{b} \varphi_{1}\left(a k_{s}\right)}{\varepsilon_{b} \varphi_{1}\left(a k_{s}\right)-\varepsilon_{s} \varphi_{1}^{(3)}\left(a k_{b}\right)},
$$

where $\varepsilon_{s}$ and $k_{s}$ are the relative dielectric function and wavenumber associated with the spherical particle. The functions $\varphi_{1}$ and $\varphi_{1}^{(3)}$ are expressed in terms of first-order spherical Bessel and Hankel functions:

$$
\varphi_{1}(z) \equiv \frac{\left[z j_{1}(z)\right]^{\prime}}{j_{1}(z)}, \quad \varphi_{1}^{(3)}(x) \equiv \frac{\left[z h_{1}(z)\right]^{\prime}}{h_{1}(z)} .
$$

It is important to underline the importance of using a frequency-dependent polarizability like that of Eq. (39) to reliably include radiation damping effects in dipole simulations. To date, radiative effects in dipole calculations were typically handled by invoking the so-called "point-scatterer" model [37-39]. Although one can arrange point scattering models to 
approximate the polarizability function of Eq. (39) for sufficiently small values of $a k_{b}$, our choice of using Mie theory to determine the polarizability seems to be more reliable for larger values of the $a k_{b}$ parameter.

Now that dipole and multipole simulations are in hand, Section $\underline{5}$ aims to illustrate the utility of the dipolar and multipolar modeling techniques by simulating a few realistic antenna designs.

\section{YAGI-UDA NANOANTENNA DESIGN WITH SPHERICAL ELEMENTS}

It has been shown that Yagi-Uda-type optical antenna designs $[\underline{10}, \underline{14}-16,18,22,23]$ can be achieved by a chain of plasmonic spheres serving as a "collector" chain and a somewhat larger sphere serving as a "reflector" element. A study of such YagiUda designs appeared as an interesting means for demonstrating the utility of the formulation derived in this work. The goal of this section is to show how the formulas developed in the previous sections provide insights into established nanoantenna designs and indicate possible design modifications. We also argue that one must be careful in specifying "optimal" nanoantenna characteristics.

Let us consider the design illustrated in Fig. 1(a) consisting of a collector made of eight silver spheres $100 \mathrm{~nm}$ in diameter combined with a silver reflector sphere $120 \mathrm{~nm}$ in diameter. The antenna is taken to be immersed in a dielectric medium of index $n_{b}=1.5$. The emitting dipole is positioned at $d=$ $30 \mathrm{~nm}$ equidistantly between the reflector particle and the collector chain. Its dipole moment is taken to be oriented perpendicular to the antenna axis. The radiation pattern of this antenna, illustrated in Fig. 1(b) for a quantum dipole emitter operating at a vacuum wavelength of $\lambda_{v}=618 \mathrm{~nm}$, clearly illustrates the desired strong directivity along the collector direction. We remark that the radiation pattern along the collector axis is relatively axisymmetric, with little radiation leaking into the backward hemisphere.

In order to quantitatively study antenna directivity, we inspired ourselves from analogous radiofrequency quantities and chose a directivity parameter of $\mathcal{D} \equiv 1_{10 \log _{10}}\left(4 \pi I_{\text {axis }} / \Gamma_{r}\right)$ $=10 \log _{10}\left(\tilde{I}_{\text {axis }} / \tilde{\Gamma}_{r}\right)$. In other words, directivity is measured in decibels of the intensity along the collector axis divided by the steradian average of total radiated power. Quantum efficiency was also calculated from its usual definition of $\eta=$ $\Gamma_{r} / \Gamma_{t}$ (for perfect emitters). All multipole simulations in this section were carried out with a multipole cutoff of $n_{\max }=10$, which proved amply sufficient for convergence in the studied configurations.

An important design property is the number of particles necessary in the collector chain. We therefore begin with multipole simulations of a Yagi-Uda design with a four-particle collector chain in Fig. 2. The principal characteristic of this antenna, as seen in Fig. 2(b), is that it becomes highly directive for vacuum wavelengths $\lambda_{v} \gtrsim 600 \mathrm{~nm}$. Because of the transverse coupling, radiation enhancements are low and even inferior to one at long wavelengths, as shown in Fig. 2(c). These low radiative enhancements are a direct consequence of the fact that this antenna design is optimized for directivity (if emission enhancements are also required, one must add a superemitter to the design (see [4,10,21]). Simulations were carried out both with and without a reflector particle. Although it is clear from Fig. 2(b) that the reflector does indeed enhance directivity, it comes at a considerable cost to the radiative decay rate and quantum efficiency, as can be seen by inspection of Figs. 2(c) and 2(d).

Next, we carried out multipole simulations for the more technically complex design of eight aligned collector particles in Fig. 3 (all other properties are the same as those in Fig. 2). The results were quite similar to those of the four-particle (a)

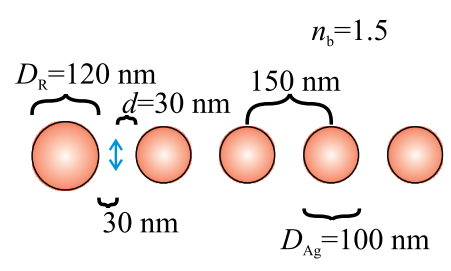

(c)

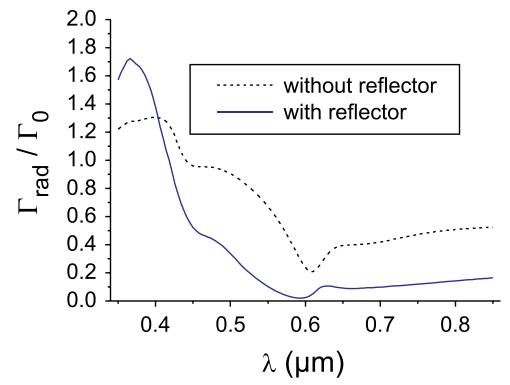

(b)

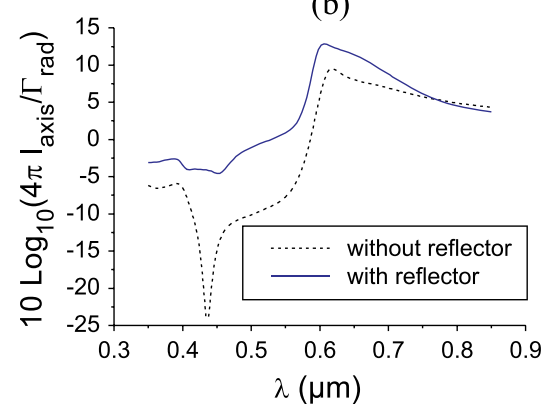

(d)

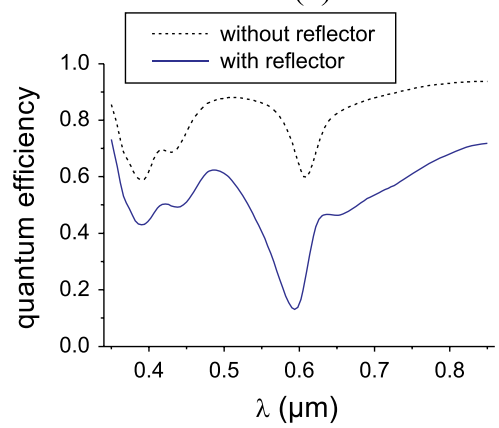

Fig. 2. (Color online) (a) Antenna schematic: four silver spheres $D_{\mathrm{Ag}}=100 \mathrm{~nm}$ in diameter with or without a $D_{R}=120 \mathrm{~nm}$ reflector particle. (b) Directivity parameter $10 \log _{10}\left(4 \pi I_{\text {axis }} / \Gamma_{r}\right)$ as a function of vacuum wavelength. (c) Radiative enhancement, $\Gamma_{r} / \Gamma_{0}$. (d) Quantum efficiency, $\eta=\Gamma_{r} / \Gamma_{\text {tot }}$. 
(a)

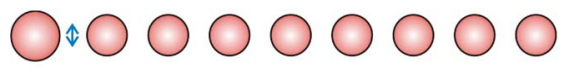

(c)

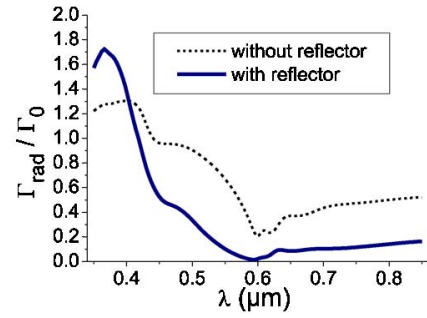

(b)

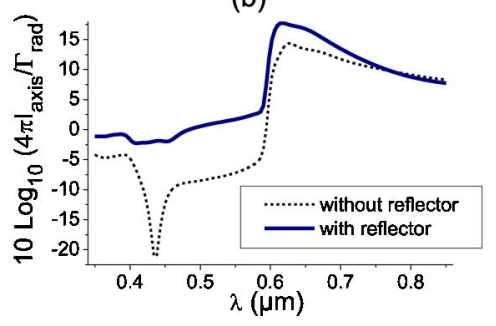

(d)

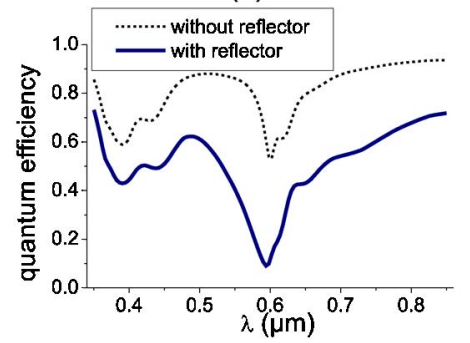

Fig. 3. (Color online) (a) Antenna schematic: same characteristics as in Fig. 2, but with an eight-particle collector array. (b) Directivity parameter $10 \log _{10}\left(4 \pi I_{\text {axis }} / \Gamma_{r}\right)$ as a function of vacuum wavelength. (c) Radiative enhancement, $\Gamma_{r} / \Gamma_{0}$. (d) Quantum efficiency, $\eta=\Gamma_{r} / \Gamma_{\text {tot }}$.

collector Yagi-Uda except for a significant increase in directivity accompanied by additional losses in quantum efficiency.

In order to better visualize the net effect of a reflector particle, the emission power along the collector axis, normalized by that of an isolated emitter, is plotted as a function of wavelength in Fig. 4 for the four- and eight-particle collector configurations studied earlier. It is clear from this figure that the reflector particle decreases the radiation intensity along the detector axis. The detrimental effect of the reflector particle could of course be decreased in this design by adopting larger spacings, $d$, between the emitter and the antenna particles, as has been done in other antenna designs in the literature. For instance, if we increased the reflector-collector separation from 60 to $100 \mathrm{~nm}$, we would arrive at a design very similar to that considered by Koenderink [10].

The quantum efficiency and radiative decay rate enhancement with different reflector-collector separations is presented in Fig. $\underline{5}$ (for the sake of simplicity, the emitter is

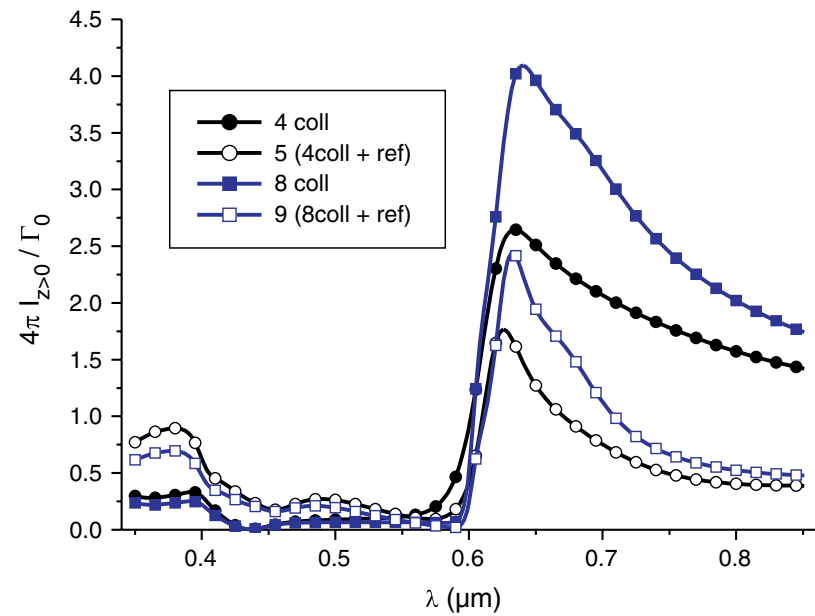

Fig. 4. (Color online) Power emitted along the collector axis for emitters $30 \mathrm{~nm}$ from the first collector particle in configurations with and without a reflector particle. Full squares, eight-particle collector without reflector; full circles, four-particle collector without reflector; open squares, eight-particle collector with reflector; open circles, four-particle collector with reflector. always taken to lie in the center of the gap). The curve labeled with diamonds is that of an emitter located at $d=50 \mathrm{~nm}$ from the collector, but without a reflector element. As we can see
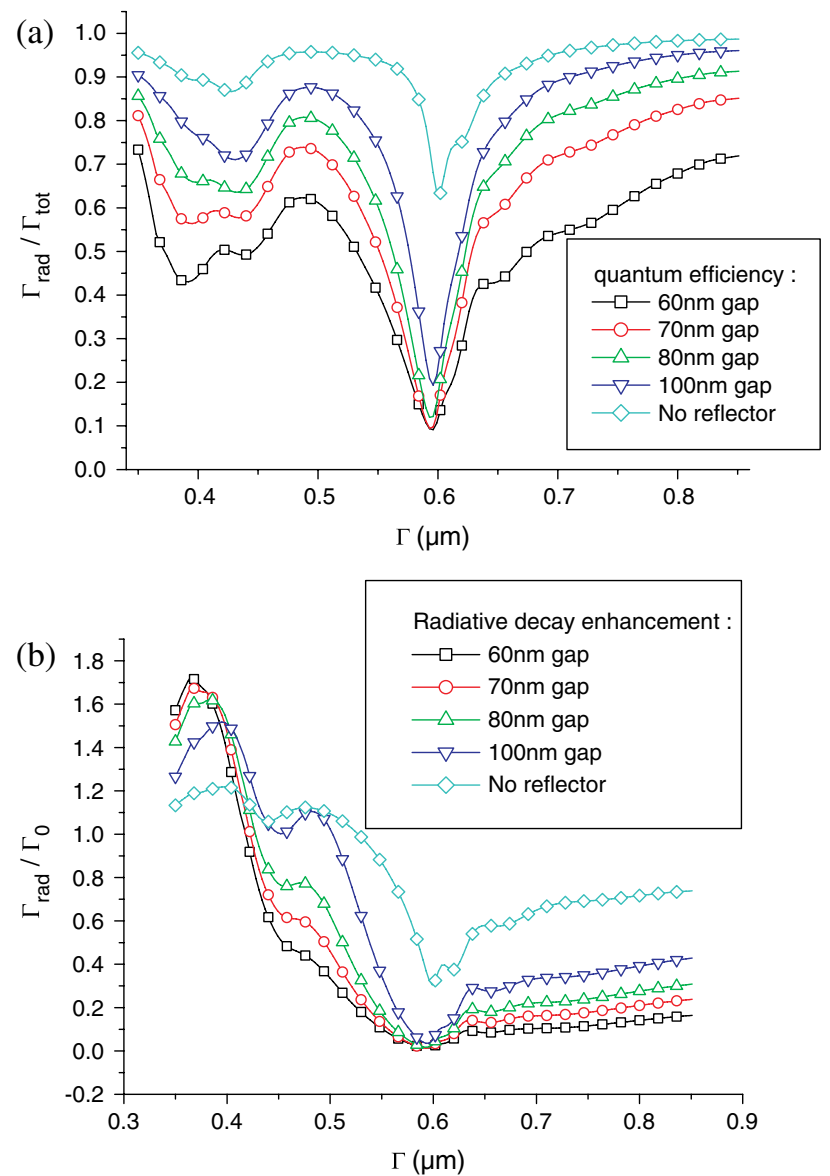

Fig. 5. (Color online) (a) Quantum efficiency and (b) radiative enhancement as functions of the wavelength for a nine-particle YagiUda with the same parameters as in Fig. 1 with varying reflectorcollector separations (emitter equidistant from reflector and first collector particles: $2 d=60,70,80,100 \mathrm{~nm}$ ). 

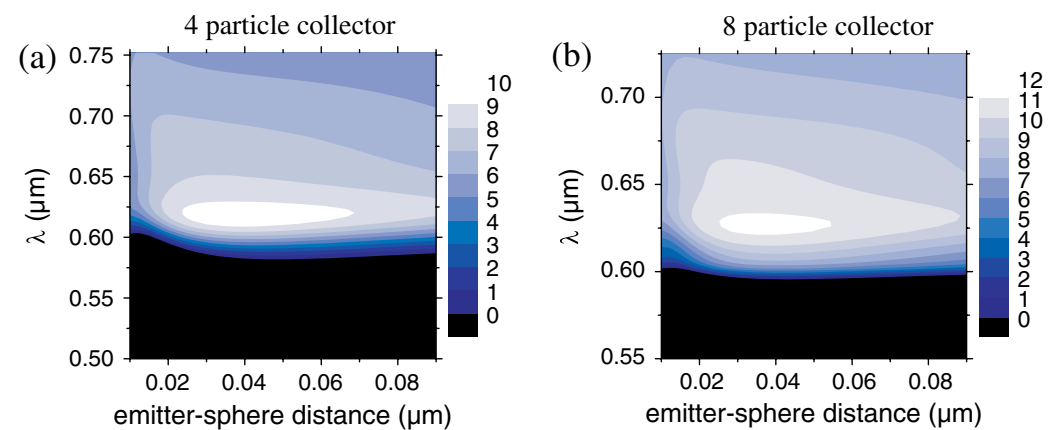

Directivity enhancement
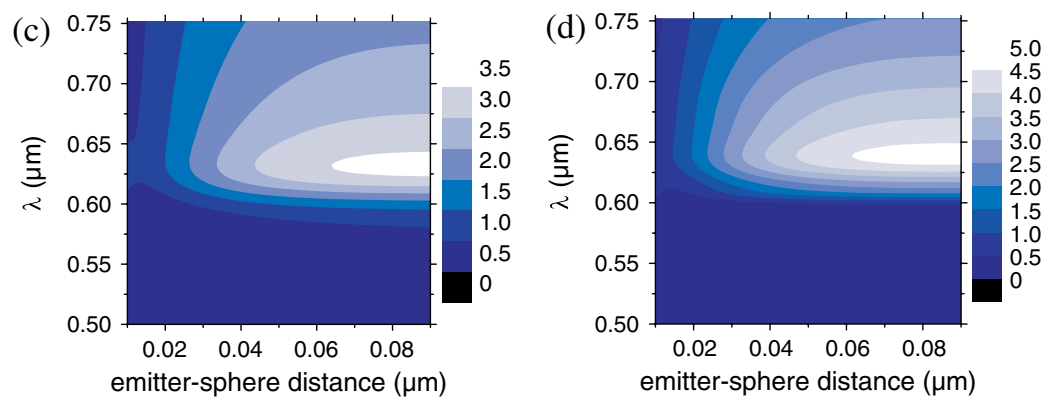

Axial power enhancement

Fig. 6. (Color online) (a), (b) Directivity, D. (c), (d) On-axis normalized radiative power enhancement as functions of wavelength and distance, $d$, of the emitter to the first collector. (a), (c) Four-particle collector, (b), (d) eight-particle collector.

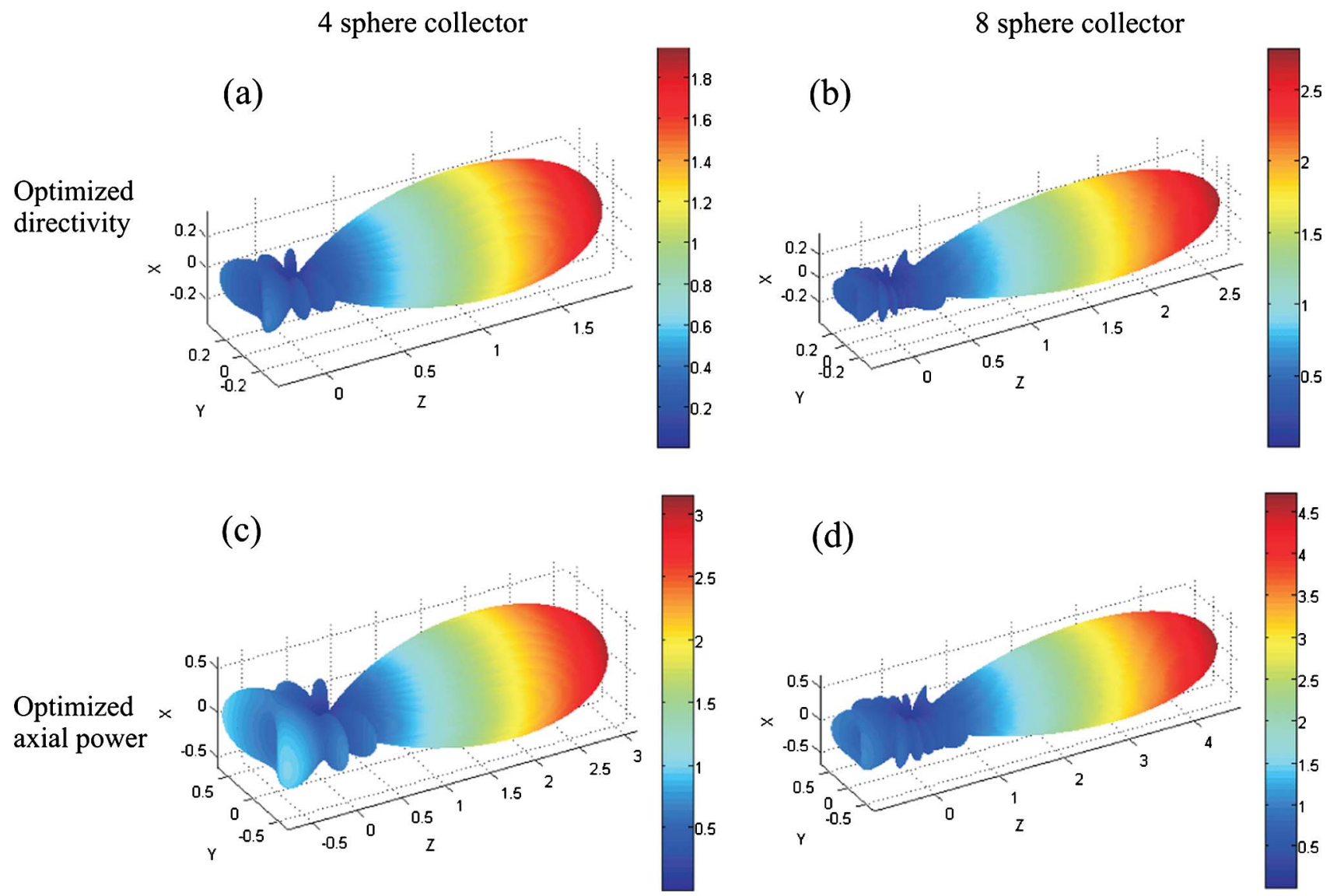

Fig. 7. (Color online) Radiation diagrams at the optimal parameters obtained from Fig. $\underline{6}$. (Irradiance is normalized such that one corresponds to the intensity of maximum free-space radiation.) Optimal directivity for a (a) four-particle collector at $\lambda=617 \mathrm{~nm}$ and emitter-collector separation of $d=40 \mathrm{~nm}$, (b) eight-particle collector at $\lambda=626 \mathrm{~nm}$ with $d=35 \mathrm{~nm}$. Optimal on-axis irradiance for a (c) four-particle collector at $\lambda=630 \mathrm{~nm}$ with $d=90 \mathrm{~nm}$, (d) eight-particle collector at $\lambda=638 \mathrm{~nm}$ with $d=85 \mathrm{~nm}$. 
from this figure, increasing gap size does indeed improve the radiative decay rates and quantum efficiency, but further gains to both quantities can still be obtained by removing the reflector. This behavior was expected and must be balanced against the advantage of a reflector element, which is to enhance emission directivity. However, the issue of directivity (and how it is defined) is directly related to our choice of reduced emitter-antenna separation distances, $d$, which we discuss below.

Let us recall that, in this work, we chose to define directivity, $\mathcal{D}$, as the radiation along the collector chain divided by the average emitted power. Optimizing $\mathcal{D}$ differs from optimizing the normalized radiated power along the collector axis, which, unlike the directivity, has contributions from the radiation enhancement factor. Directivity enhancement and on-axis power enhancement as functions of wavelength and emittercollector distance are plotted in Fig. 6 for both the four- and eight-particle collector antennas (without reflectors). As we can see in Fig. 6, optimizing directivity favors somewhat smaller emitter-collector distances, $d$, than does an optimization of the on-axis power enhancement, which explains the choices in this work. One can perhaps better visualize the situation by examining the radiation diagrams of the chains at their optimum parameters for directivity and power respectively for both four- and eight-particle chains, which are presented in Fig. 7.

Another design consideration that seems appropriate to mention is that of orienting the dipole emitter perpendicular to the antenna chain. As can be seen from Fig. $\underline{8}$, the directivity drops rapidly with variation of the emitter axis from the perpendicular orientation (falling by $\simeq 3 \mathrm{~dB}$ at $\simeq 10^{\circ}$ from a perpendicular orientation). For randomly oriented dipoles, this poses significant limitations to antenna properties. However, dipole emissions perpendicular to the collector axis can be privileged by an on-axis superemitter around the quantum emitter $[\underline{10}, \underline{20}, \underline{21}]$ or off-axis longitudinal couplings of an emitter to an antenna particle $[\underline{16}, \underline{19}, \underline{23}]$. In such cases, the longitudinal couplings induce strong decay rate enhancements

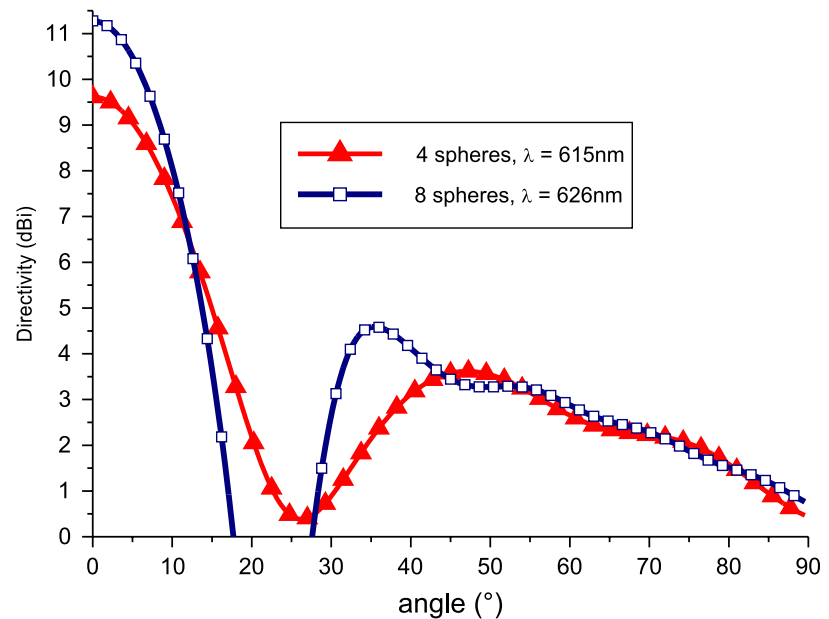

Fig. 8. (Color online) Directivity, in decibels, plotted as a function of the angle of the emitter with the direction perpendicular to the collector chain for a four- and eight-particle collector chain (without reflector) and emitter-collector separations of $d=30 \mathrm{~nm}$ (respective operating wavelengths of $\lambda=615 \mathrm{~nm}$ and $\lambda=626 \mathrm{~nm}$ selected respectively for high directivities).

that can largely outweigh the relatively modest decay rate damping resulting from small emitter-collector separations.

The last issue that we wish to address in this section is the feasibility of purely electric dipole modeling of these antennas. Although it has already been pointed out that multipoles are quite important in quantitative studies of the longitudinal couplings of "superemitter" designs, the Yagi-Uda design relies on the much weaker transverse couplings, and one expects the dipolar model developed in Section $\underline{4}$ to be approximately valid in this case.

The results obtained using the coupled dipole formulas of Subsection 4.A coupled with the Mie-based polarizability of Subsection $\overline{4 . \mathrm{B}}$ are compared with multipole calculations in Fig. 9 for an eight-particle collector antenna. Although there are significant differences between the dipolar and multipolar results at shorter wavelengths, the coupled dipole calculations correspond rather well to the quasi-exact results at
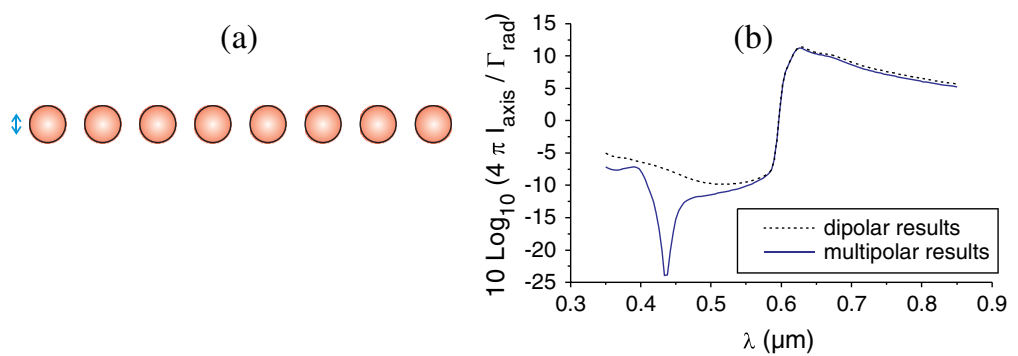

(c)

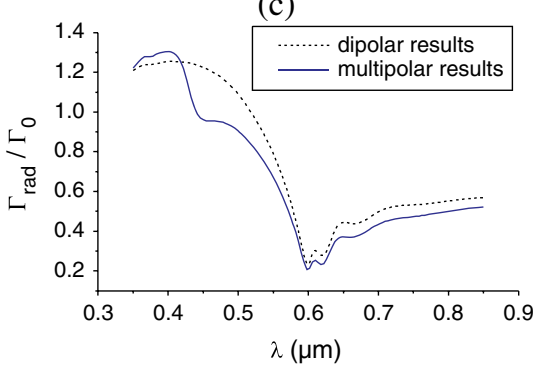

(d)

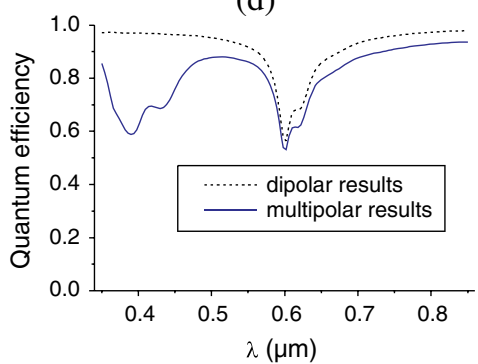

Fig. 9. (Color online) (a) Comparison between coupled electric dipole results and multipole calculations for an eight-particle collector array antenna. (b) Directivity parameter $10 \log _{10}\left(4 \pi I_{\text {axis }} / \Gamma_{r}\right)$. (c) Radiative enhancement, $\Gamma_{r} / \Gamma_{0}$. (d) Quantum efficiency, $\eta=\Gamma_{r} / \Gamma_{\text {tot }}$. 
the larger wavelengths, i.e., $\lambda_{\mathrm{v}} \gtrsim 600 \mathrm{~nm}$, for which the antenna is designed. Of course, there are some differences between the coupled dipole calculations and the exact multipole result at all wavelengths, but these can largely be attributed to the proximity of the emitter to plasmonic antenna particles, a situation that excites higher-order modes in neighboring plasmonic particles.

\section{CONCLUSIONS}

An important result of this work was to derive analytic multipole formulas for calculating the quantum emission properties of nanoantennas in a manner that avoided numerical integrations of energy flow. The gain in speed and accuracy provides significant advantages to nanoantenna design. We also derived new analytical formulas in the coupled dipole approximation, which permit the integration-free calculation of quantum decay rates of quantum emitters in Yagi-Uda-type designs.

In Section 5, we performed an in-depth study of the radiative and directive properties of Yagi-Uda antennas. We studied therein a variety of antenna design properties, including the number of particles in the collector chain, the quantum emitter's orientation and position, and the utility of a reflector particle in optical Yagi-Uda antennas. Concerning this last point, we showed that the improvements in beam directivity provided by a reflector particle come at a considerable expense to the radiative decay rate and quantum yield. We propose that reflector-free antennas may prove to be an interesting alternative design.

\section{APPENDIX A}

\section{VSHs}

VSHs are described in a number of works [35,40-42], but the notations and definitions vary with authors. They form a complete orthogonal basis set for describing the angular variations of any vector field. We define the spherical VSHs as follows:

$$
\begin{aligned}
\mathbf{Y}_{n m}(\theta, \phi) & \equiv \hat{\mathbf{r}} Y_{n m}(\theta, \phi), \\
\mathbf{Z}_{n m}(\theta, \phi) & \equiv \frac{r \nabla Y_{n m}(\theta, \phi)}{\sqrt{n(n+1)},} \\
\mathbf{X}_{n m}(\theta, \phi) & \equiv \mathbf{Z}_{n m}(\theta, \phi) \times \widehat{\mathbf{r}} .
\end{aligned}
$$

All VSHs are mutually orthogonal in the sense that taking $\mathbf{W}_{n m}^{(i)}(i=0,1,2)$ to respectively denote the VSHs $\mathbf{Y}_{n m}, \mathbf{X}_{n m}$, or $\mathbf{Z}_{n m}$, we have

$$
\left\langle\mathbf{W}_{n m}^{(i)} \mid \mathbf{W}_{\nu \mu}^{(j)}\right\rangle \equiv(-)^{m} \int_{0}^{4 \pi} \mathbf{W}_{n,-m}^{(i)} \cdot \mathbf{W}_{\nu \mu}^{(j)} \mathrm{d} \Omega=\delta_{i j} \delta_{n \nu} \delta_{m \mu} .
$$

\section{VPWs}

The VPWs are often referred to as regular spherical vector waves, $R g\left\{\mathbf{M}_{n m}(k \mathbf{r})\right\}$ and $R g\left\{\mathbf{N}_{n m}(k \mathbf{r})\right\}$ [35], which are respectively the direct space representations of the VPWs $\left\langle\mathbf{r} \mid \Psi_{q=1, n, m}\right\rangle$ and $\left\langle\mathbf{r} \mid \Psi_{q=2, n, m}\right\rangle$. Their analytical expressions are given by

$$
\begin{aligned}
\left\langle\Psi_{1, n, m} \mid \mathbf{r}\right\rangle \equiv & R g\left\{\tilde{\mathbf{M}}_{n, m}(k \mathbf{r})\right\} \equiv(-)^{m} j_{n}(k r) \mathbf{X}_{n,-m}(\hat{\mathbf{r}}) \\
\left\langle\Psi_{2, n, m} \mid \mathbf{r}\right\rangle \equiv & R g\left\{\tilde{\mathbf{N}}_{n m}(k \mathbf{r})\right\}, \quad n=1,2, \ldots \infty, \quad m=-n, \ldots, n \\
\equiv & \frac{(-)^{m}}{k r}\left\{\sqrt{n(n+1)} j_{n}(k r) \hat{\mathbf{r}} Y_{n,-m}(\hat{\mathbf{r}})\right. \\
& \left.+\left[k r j_{n}(k r)\right]^{\prime} \mathbf{Z}_{n,-m}(\widehat{\mathbf{r}})\right\} .
\end{aligned}
$$

The total field in the antenna problem satisfies outgoing boundary conditions. Consequently, when evaluating the total fields, e.g., in Eq. (19), the fields were developed in terms of VPWs satisfying the outgoing boundary conditions, traditionally labeled $\mathbf{M}_{n m}$ and $\mathbf{N}_{n m}$ [35], which are analogous to the regular waves of Eq. (A5) with the spherical Bessel functions being replaced by spherical Hankel functions:

$$
\begin{aligned}
& \mathbf{N}_{n m}(k \mathbf{r}) \equiv \frac{1}{k r}\left\{\sqrt{n(n+1)} h_{n}(k r) \hat{\mathbf{r}} Y_{n m}(\hat{\mathbf{r}})+\left[k r h_{n}(k r)\right]^{\prime} \mathbf{Z}_{n m}(\widehat{\mathbf{r}})\right\}, \\
& \mathbf{M}_{n m}(k \mathbf{r}) \equiv h_{n}(k r) \mathbf{X}_{n m}(\hat{\mathbf{r}}) .
\end{aligned}
$$

\section{ACKNOWLEDGMENTS}

This research is partly funded by the French Agence Nationale de la Recherche under contract ANR-07-NANO-006-03 ANTARES.

\section{REFERENCES}

1. H. Metiu, "Surface enhanced spectroscopy," Prog. Surf. Sci. 17, 153-320 (1984).

2. H. Tamaru, H. Kuwata, H. T. Miyazaki, and K. Miyano, "Resonant light scattering from individual Ag nanoparticles and particle pairs," Appl. Phys. Lett. 80, 1826-1828 (2002).

3. M. Thomas, J.-J. Greffet, R. Carminati, and J. R. Arias-Gonzalez, "Single-molecule spontaneous emission close to absorbing nanostructures," Appl. Phys. Lett. 85, 3863-3865 (2004).

4. J. N. Farahani, D. W. Pohl, H.-J. Eisler, and B. Hecht, "Single quantum dot coupled to a scanning optical antenna: a tunable superemitter," Phys. Rev. Lett. 95, 017402 (2005).

5. P. Anger, P. Bharadwaj, and L. Novotny, "Enhancement and quenching of single-molecule fluorescence," Phys. Rev. Lett. 96, 113002 (2006).

6. R. Carminati, J.-J. Greffet, C. Henkel, and J. Vigoureux, "Radiative and non-radiative decay of single molecule close to a metallic nanoparticle," Opt. Commun. 261, 368-375 (2006).

7. H. Mertens, A. F. Koenderink, and A. Polman, "Plasmonenhanced luminescence near noble-metal nanospheres: comparison of exact theory and an improved Gersten and Nitzan model," Phys. Rev. B 76, 115123 (2007).

8. L. Rogobete, F. Kaminski, M. Agio, and V. Sandoghdar, "Design of plasmonic nanoantennae for enhancing spontaneous emission," Opt. Lett. 32, 1623-1625 (2007).

9. A. Kinkhabwala, Z. Yu, S. Fan, Y. Avlasevich, K. Mullen, and W. E. Moerner, "Large single-molecule fluorescence enhancements produced by a bowtie nanoantenna," Nat. Photon. 3, 654-657 (2009).

10. A. Koenderink, "Plasmon nanoparticle array waveguides for single photon and single plasmon sources," Nano Lett. 9, 4228-4233 (2009).

11. J. W. Liaw, C. S. Chen, and J. H. Chen, "Enhancement or quenching effect of metallic nanodimer on spontaneous emission," J. Quant. Spectrosc. Radiat. Transfer 111, 454-465 (2010).

12. H. Aouani, O. Mahboud, N. Bonod, E. Devaux, E. Popov, H. Rigneault, T. W. Ebbesen, and J. Wenger, "Bright unidirectional fluorescence emission of molecules in a nanoaperture with plasmonic corrugations," Nano Lett. 11, 637-644 (2011).

13. H. Gersen, M. F. Garcia-Parajo, L. Novotny, J. A. Veerman, L. Kuipers, and N. F. van Hulst, "Influencing the angular emission of a single molecule," Phys. Rev. Lett. 85, 5312-5315 (2000). 
14. H. F. Hofman, T. Kosako, and Y. Kadoya, "Design parameters for a nano-optical Yagi-Uda antenna," New J. Phys. 9, 217 (2007).

15. J. Li, A. Salandrino, and N. Engheta, "Shaping light beams in the nanometer scale: a Yagi-Uda nanoantenna in the optical domain," Phys. Rev. B 76, 245403 (2007).

16. T. H. Taminiau, F. D. Stefani, and N. F. van Hulst, "Enhanced directional excitation and emission of single emitters by a nano-optical Yagi-Uda antenna," Opt. Express 16, 10858-10866 (2008).

17. T. H. Taminiau, F. D. Stefani, F. B. Segerink, and N. F. van Hulst, "Optical antennas direct single-molecule emission," Nat. Photon. 2, 234-237 (2008).

18. D. Gérard, A. Devilez, H. Aouani, B. Stout, N. Bonod, J. Wenger, E. Popov, and H. Rigneault, "Efficient excitation and collection of single-molecule fluorescence close to a dielectric microsphere,” J. Opt. Soc. Am. B 26, 1473-1478 (2009).

19. T. Pakizeh and M. Kall, "Unidirectional ultracompact optical antennas," Nano Lett. 9, 2343-2349 (2009).

20. A. Devilez, B. Stout, and N. Bonod, "Compact metallo-dielectric optical antenna for ultra directional and enhanced radiative emission," ACS Nano. 4, 3390-3396 (2010).

21. N. Bonod, A. Devilez, B. Rolly, S. Bidault, and B. Stout, "Ultracompact and unidirectional metallic antennas," Phys. Rev. B 82, 115429 (2010).

22. T. Kosako, Y. Kadoya, and H. F. Hofmann, "Directional control of light by a nano-optical Yagi-Uda antenna," Nat. Photon. 4, 312-315 (2010).

23. A. Curto, G. Volpe, T. Taminiau, M. Kreuzer, R. Quidant, and N. van Hulst, "Unidirectional emission of a quantum dot coupled to a nanoantenna," Science 329, 930-933 (2010).

24. M. Kerker, D.-S. Wang, and H. Chew, "Surface enhanced Raman scattering (SERS) by molecules adsorbed at spherical particles: errata,” Appl. Opt. 19, 4159-4174 (1980).

25. R. Ruppin, "Decay of an excited molecule near a small metal sphere,” J. Chem. Phys. 76, 1681-1684 (1982).

26. Y. Kim, P. Leung, and T. George, "Classical decay rates for molecules in the presence of a spherical surface: a complete treatment," Surf. Sci. 195, 1-14 (1988).

27. G. C. des Francs, A. Bouhelier, E. Finot, J. Weeber, A. Dereux, and E. Dujardin, "Fluorescence relaxation in the near-field of a mesoscopic metallic particle: distance dependence and role of plasmon modes," Opt. Express 16, 17654-17666 (2008).

28. W. C. Chew, Waves and Fields in Inhomogeneous Media (IEEE, 1990).

29. B. Stout, J.-C. Auger, and J. Lafait, "A transfer matrix approach to local field calculations in multiple scattering problems," J. Mod. Opt. 49, 2129-2152 (2002).

30. B. Stout, J. Auger, and A. Devilez, "Recursive T matrix algorithm for resonant multiple scattering: applications to localized plasmon excitations,” J. Opt. Soc. Am. A 25, 2549-2557 (2008).

31. J.-C. Auger, V. Martinez, and B. Stout, "Absorption and scattering properties of dense ensembles of non-spherical particles," J. Opt. Soc. Am. A 24, 3508-3516 (2007).

32. L. Novotny and B. Hecht, "Light emission and optical interactions in nanoscale environments," in Principles of Nano-Optics (Cambridge University, 2006), pp. 250-303.

33. L. Tsang and J. A. Kong, "Multiple scattering of electromagnetic waves by random distributions of discrete scatterers with coherent potential and quantum mechanical formalism,” J. Appl. Phys. 51, 3465-3485 (1980).

34. M. Lax, "Multiple scattering of waves," Rev. Mod. Phys. 23, 287-310 (1951).

35. L. Tsang, J. A. Kong, and R. T. Shin, Theory of Microwave Remote Sensing (Wiley, 1985).

36. C. F. Bohren and D. R. Huffman, Absorption and Scattering of Light by Small Particles (Wiley, 1983).

37. Y. P. Pellegrini, B. Stout, and P. Thibaudeau, "Off-shell meanfield electromagnetic $T$-matrix of finite size spheres and fuzzy scatterers,” J. Phys. Condens. Matter 9, 177-191 (1997).

38. P. de Vries, D. V. van Coevorden, and A. Lagendijk, "Point scatterers for classical waves,” Rev. Mod. Phys. 70, 447-466 (1998).

39. A. Lagendijk and B. Tiggelin, "Resonant multiple scattering of light," Phys. Rep. 270, 143-215 (1996).

40. A. R. Edmonds, Angular Momentum in Quantum Mechanics (Princeton University, 1960).

41. C. Cohen-Tannoudji, J. Dupont-Roc, and G. Grynberg, Photons \& Atoms: Introduction to Quantum Electrodynamics (Wiley, 1997).

42. J. D. Jackson, Classical Electrodynamics, 3rd ed. (Wiley, 1999). 